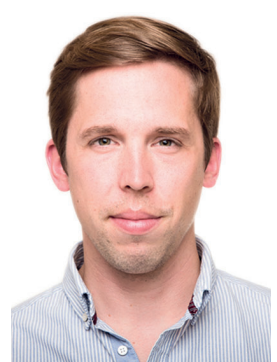

Matthias Raspe

Koordination «PneumoCampus»

\section{Familienanamnese bei der Diagnosestellung, Therapiesteckbriefe und Weiterbildungsabschnitte}

\begin{abstract}
In dieser Ausgabe des PneumoCampus haben wir wieder einige interessante Beiträge für Sie zusammengestellt: Testen Sie Ihre Diagnosefähigkeiten anhand des dargestellten Falls und überprüfen Sie anschließend Ihre Vermutung auf der KARGER-KOMPASS-PNEUMOLOGIEWebseite.

In unserer Serie zu den aktuellen Therapiemaßnahmen beim nicht kleinzelligen Lungenkarzinom widmen wir uns dieses Mal dem Tumorstadium IV mit Treibermutation. Der «Überlebenstipp» informiert Sie über die verschiedenen Abschnitte der fachärztlichen Weiterbildung und verrät Ihnen, was Sie dabei beachten sollten.
\end{abstract}

\section{Blickdiagnosen \\ Welche Diagnose vermuten Sie?}

Der 49-jährige Patient stellte sich aufgrund einer seit etwa 3 Monaten rasch zunehmenden Atemnot über die Notaufnahme unserer Klinik vor. Die Krankenhauseinweisung hatte ein niedergelassener Pneumologe veranlasst, der dem Patienten den kürzlich erhobenen Befund C mitgegeben hatte. Der Patient war bis zum Aufnahmetag voll berufstätig. Er berichtete außerdem eine stark ausgeprägte Tagesmüdigkeit, eine schlechte Schlafqualität, da er aufgrund von Luftnot im Liegen nachts immer wieder aufstehen müsse, und eine verminderte körperliche Belastbarkeit. Ansonsten war der Patient gesund und ohne relevante Vorerkrankungen. Es bestand ein aktiver Nikotinkonsum. Lähmungen wurden nicht direkt beschrieben, allerdings hätten ihn Nahestehende bereits häufiger auf sein Gangbild angesprochen. Bezüglich der Familienanamnese berichtete er, dass sein Bruder aufgrund ähnlicher Beschwerden mit einer «Atemmaske» versorgt worden sei. In der Notaufnahme wurde eine Computertomographie des Thoraxangefordert; Abbildung A zeigt ein koronares Schnittbild dieser Untersuchung. Abbildung B zeigt den spirometrischen Befund des Patienten.

\section{Auflösung unter:}

www.karger.com/blickdiagnose-kkp-5-2019

Kontaktadresse: Dr. Matthias Raspe, Medizinische Klinik mit Schwerpunkt Infektiologie und Pneumologie, Charité - Universitätsmedizin Berlin, Augustenburger Platz 1, 13353 Berlin, Deutschland, matthias.raspe@charite.de

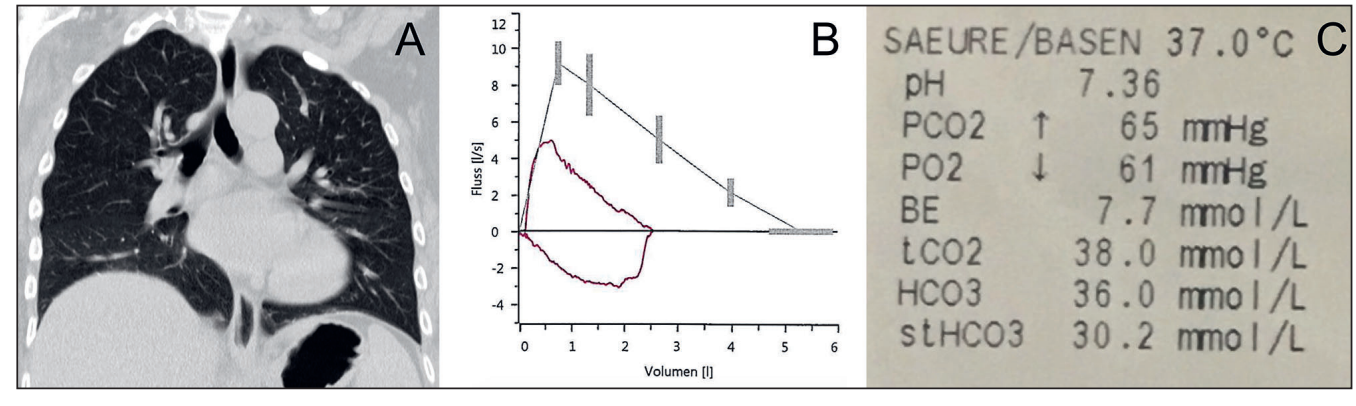

Bildbefund A mit freundlicher Genehmigung von PD Dr. med. C. Althoff, Leitung Interventionsradiologie, Charité Campus Mitte, Institut und Klinik für Radiologie CCM und CVK, Charité - Universitätsmedizin Berlin; Bildbefunde B und C stammen aus der eigenen Klinik.

\title{
KARGER
}

(c) 2019 S. Karger GmbH, Freiburg 


\section{Steckbriefe zur aktuellen Therapie des nicht kleinzelligen Lungenkarzinoms Stadium IV mit Treibermutation}

Das Lungenkarzinom ist bei Frauen der dritt- und bei Männern der zweithäufigste maligne Tumor in den deutschsprachigen Ländern mit einer hohen tumorspezifischen Mortalität (bei Männern Platz 1 und bei Frauen Platz 3 in Deutschland). Erfreulicherweise haben sich im letzten Jahrzehnt Therapieoptionen und Prognose durch Zulassung vieler neuer Präparate aus dem Bereich der TyrokinaseInhibitor und Immuntherapie deutlich verbessert - hiervon profitieren im Wesentlichen Patienten in fortgeschrittenen Krankheitsstadien.

In pneumologischen Abteilungen sind Patienten mit Lungenkarzinomen häufig. Andererseits erfordert die Begleitung dieser Patienten viel Erfahrung und Wissen um den aktuellen State-of-the-art in Diagnostik und Therapie. Gerade jüngere Kolleginnen und Kollegen werden hier vor eine große Aufgabe gestellt. Mit dieser 4-teiligen Serie wollen wir einen praxisnahen und übersichtlichen Beitrag zur aktuellen Therapie des nicht kleinzelligen Lungenkarzinoms (non-small cell lung carcinoma, NSCLC) geben. Im nun dritten Teil soll es um die Therapie des NSCLC im Stadium IV mit Treibermutation gehen.

Die Klassifikation des NSCLC erfolgt aktuell nach IASLC (International Association for the Study of Lung Cancer)/UICC (Union for International Cancer Controll) Version 8 [1]. Die folgende Tabelle gibt einen Überblick über die Einteilung des Stadiums IV. Das Muster der Fernmetastasierung wird dabei wie folgt unterschieden: «M1a» bezeichnet den Befall der kontralateralen Lunge, der Pleura, einen malignen Pleurerguss oder einen malignen Perikarderguss. Neu aufgenommen in die Version 8 der Staging-Klassifikation wurde das Stadium «M1b», dasfüreine sogenannteoligometastasierte Erkrankung steht. Darunter wird eine singuläre Metastase in einem extrathorakalen Organ (wie z.B. dem Hirn) verstanden. «M1c» steht für mehrere Fernmetastasen in einem oder mehreren Organen.

Tumorstadium III des NSCLC nach IASLC/UICC Version 8

\begin{tabular}{llll}
\hline Stadium & Primärtumor & Lymphknoten & Fernmetastasen \\
\hline IVA & jedes T & jedes N & M1a \\
& jedes T & jedes N & M1b \\
\hline IVB & jedes T & jedes N & M1c \\
\hline
\end{tabular}

Die rasante Entwicklung der molekularen Charakterisierung des Lungenkarzinoms und zielgerichteter Therapien für sogenannte Treibermutationen haben für einen kleinen Teil der Patienten im Stadium IV äußerst effektive Therapieoptionen eröffnet, die in der Regel mit einer deutlich besseren Prognose einhergehen. Heutzutage muss eine molekularpathologische Analyse des Tumorgewebes auf die wesentlichen Treibermutationen (EGFR, ALK, ROS1 und BRAF), für die zielgerichtete Therapien aus der Klasse der Tyrosinkinase-Inhibitoren (TKI) zugelassen sind, bei allen Patienten mit NSCLC/Adenokarzinom und Patienten mit NSCLC/Plattenepithelkarzinom mit wenig (in der Regel <10 Packungsjahre) oder keinem
Nikotinkonsum in der Vorgeschichte vor Therapiebeginn erfolgen (neben einer PD-L1-Immunhistochemie). Idealerweise findet eine molekulare Charakterisierung des Tumors noch wesentlich tiefer mit einer zusätzlichen Suche nach seltenen therapierelevanten Mutationen statt, wie es z.B. im «Nationalen Netzwerk Genomische Medizin» (nNGM, www.nngm.de) angeboten wird. TKI überzeugen mit einer für Patienten im Vergleich zur konventionellen Chemotherapie deutlich angenehmeren oralen Einnahme, einem überwiegend besser tolerier- und beherrschbaren Nebenwirkungsprofil und vor allem einer deutlich überlegenen Krankheitskontrolle. Für alle TKI müssen die spezifischen Einnahmehinweise und $\mathrm{Ne}$ benwirkungen beachtet und die Patienten gut darauf vorbereitet werden. In den Fachinformationen der Hersteller werden konkrete Maßnahmen zum Umgang und Verhalten bei Nebenwirkungen beschrieben. Auch für Patienten in reduziertem Allgemeinzustand sollte, sofern möglich, eine TKI-Therapie erwogen werden (Performance-Status der Eastern Cooperative Oncology Group (ECOG) 2-4), vor allem dann, wenn der Tumor maßgeblich für den eingeschränkten Allgemeinzustand verantwortlich ist. In vielen TKI-Studien ist der Überlebensvorteil durch Crossover-Effekte gering ausgeprägt bzw. nicht vorhanden. Nach Auswertungen aus dem nNGM betrug das mediane Überleben unter Therapie mit einem EGFR-TKI 31,5 Monate gegenüber 9,6 Monaten unter Chemotherapie [2]. Die Abbildung auf der folgenden Seite zeigt den hausinternen Therapiealgorithmus aus der Klinik m.S. Infektiologie und Pneumologie der Charité Berlin zur Therapie des NSCLC im Stadium IV.

\section{NSCLC mit EGFR-Mutation}

Bei einem großen Teil der NSCLC ist eine prgnostisch ungünstige EGFR-Überexpression nachzuweisen. Aber erst die Entdeckung von aktivierenden EGFR-Mutationen (bei etwa 15\% der Adenokarzinome) 2004 brachte eine schlüssige Erklärung dafür, weshalb bislang nur ein geringer Teil der Patienten von einer Hemmung der EGFR-vermittelten Signaltransduktion profitiert hatte. Die Mutationen im EGFR-Gen betreffen die Exone 18-22 der Tyrosinkinase-Domäne. Die häufigsten Mutationen sind Deletionen im Exon 19 und Punktmutationen im Exon 21 (L858R), wobei die Erstgenannte prognostisch am günstigsten ist. Als EGFR-TKI sind mittlerweile 3 Generationen zugelassen: Die Erstlinien-TKI Erlotinib (jeweils zulassungsrelevante Studien angegeben; [3]) und Gefitinib [4], die Zweitlinien-TKI Afatinib [5] oder Dacomitinib [6] und der Drittlinien-TKI Osimertinib [7]. Aufgrund seiner überlegenen Wirksamkeit (auch bei ZNS-Metastasen) und Dauer des Therapieansprechens wird Osimertinib mittlerweile als Erstlinienpräparat bei Nachweis einer aktivierenden EGFR-Mutation bevorzugt. In der für die Erstlinie zulassungsrelevanten FLAURA-Studie wurde Osimertinib mit den Erstlinien-TKI Erlotinib und Gefitinib verglichen. Da für Afatinib in einer gepoolten Analyse der LUX-Lung-3- und LUX-Lung-6-Studien für Exon-19-Deletionen ein verbessertes Gesamtüberleben gegenüber Chemotherapie belegt wurde, kann Afatinib weiterhin in der Erstlinie bei dieser Patientengruppe eingesetzt werden. Osimerti- 


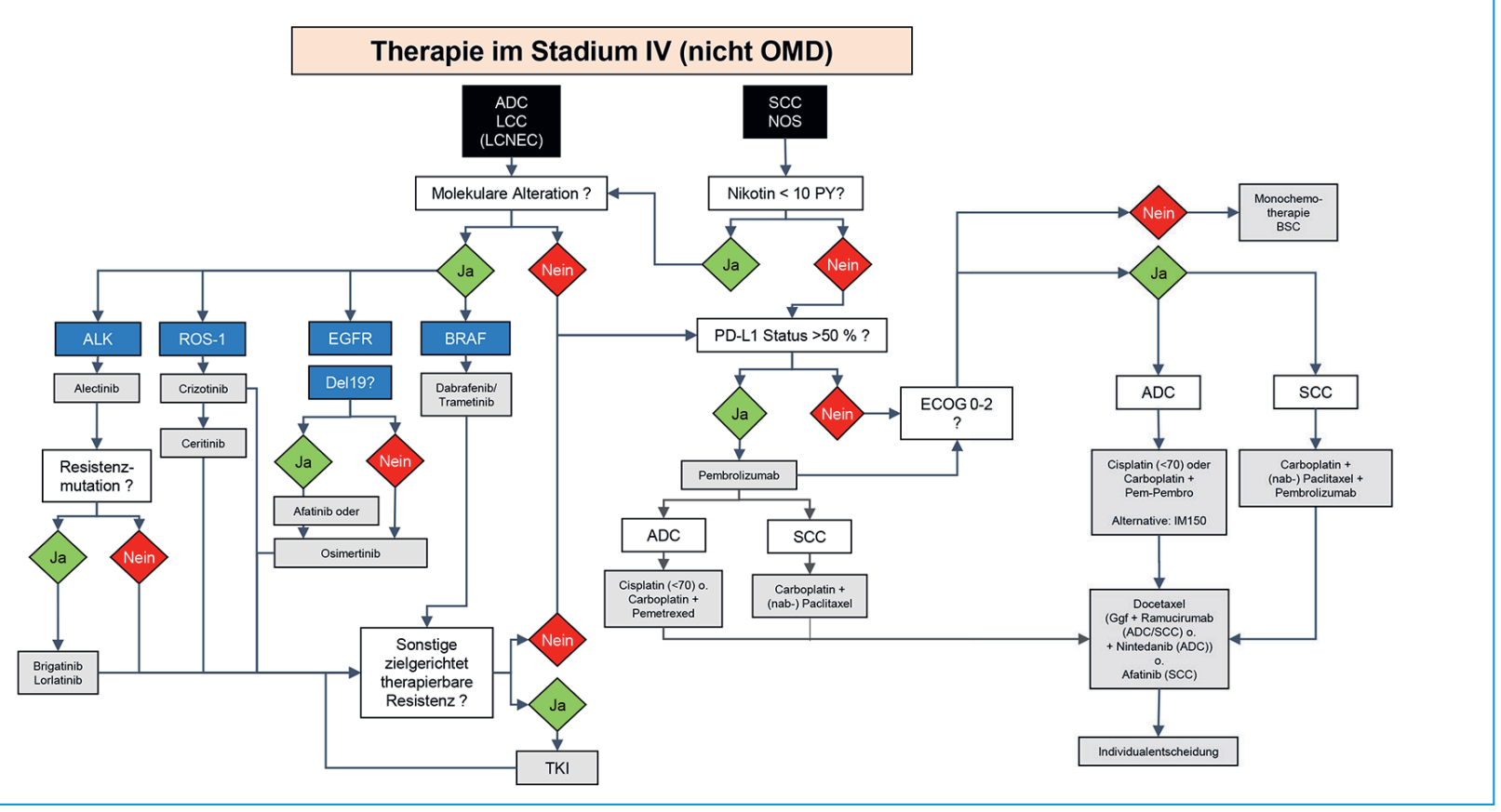

(Hausinterner) Therapiealgorithmus des NSCLC im Stadium IV. OMD, oligometastasierte Erkrankung; ADC, Adenokarzinom; SCC, Plattenepithelkarzinom; NOS, not otherwise specified (NSCLC ohne klare Zuordnung ADC oder SCC); LCC, großzelliges Karzinom; LCNEC, großzelliges neuroendokrines Karzinom; Del19, Exon-19-Deletion; TKI, Tyrokinase-Inhibitor. @ Nikolaj Frost 2019.

nib ist auch bei Entwicklung einer T790M-Resistenzmutation unter EGFR-TKI der 1. oder 2. Generation TKI der Wahl. Alle TKI haben ein charakteristisches Nebenwirkungsprofil, das vor allem die Haut/Nägel und den Magen-Darm-Trakt betreffen. Aufgrund seiner selektiveren Bindung an den mutierten EGFR-Rezeptor ist Osimertinib nebenwirkungsärmer als TKI der beiden vorherigen Generationen.

\section{ALK-Translokationen}

Translokationen im ALK-Gen betreffen etwa 3-5\% der Patienten mit Adenokarzinom. Auch für ALK-Translokationen sind mittlerweile mehrere Generationen von TKI zugelassen. Zur 1. Generation von ALK-TKI zählt Crizotinib [8], zur 2. Generation zählen Alectinib [9] und Ceritinib [10] und zur 3. Generation unter anderem Brigatinib [11] und Lorlatinib [12]. Zur Erstlinientherapie des ALK-mutierten NSCLC wird aktuell Alectinib empfohlen, dass im Vergleich zu Crizotinib eine deutlich bessere Wirksamkeit bei Hirnmetastasen, eine bessere Verträglichkeit und ein verlängertes progressionsfreies Überleben (progression-free survival, PFS) aufweisen kann. Für Alectinib sind neben den typischen Nebenwirkungen an Haut und Gastrointestinaltrakt als sehr häufige unerwünschte Befunde Blutbildveränderungen, Leberstoffwechselstörungen, Myalgien und Ödeme beschrieben. Ceritinib ist ebenfalls in der Erstlinie zugelassen, wurde aber in der zulassungsrelevanten Studie nicht mit Crizotinib, sondern mit einer platinbasierten Chemotherapie verglichen. Außerdem ist die gastrointestinale Verträglichkeit dieses TKI vergleichsweise schlecht. Drittlinien-ALKTKI sind nur für Folgelinien zugelassen. Dadurch dass der Tumor ständig weiter mutiert, sind Fälle beschrieben, bei denen im Verlauf neue therapierbare Mutationen (wieder-)auftreten. Bei Krankheitsprogress sollten also erneute Probenentnahmen und
Mutationsanalysen Standard sein (mit der Frage nach Resistenzmutationen und neuen Targets).

\section{ROS1-Translokationen}

ROS1-Translokationen sind selten und betreffen etwa 1-2\% der Adenokarzinome. Die bisherige Erstlinientherapie der Wahl erfolgt mit Crizotinib [13]. Wirksamkeit scheinen auch die TKI Ceritinib, Cabozantinib und Lorlatinib zu haben.

\section{BRAF-Mutationen}

BRAF-Mutationen sind ebenfalls selten. Unter innen ist die V600EPunktmutation mit etwa 1-2\% der Adenokarzinome die häufigste. Zugelassen ist hier eine Kombination aus dem BRAF-Inhinitor Dabrafenib und MEK-Inhibitor Trametinib [14].

\section{Literatur}

1 Goldstraw P, Chansky K, Crowley J, et al.: J Thorac Oncol 2016;11:39-51.

2 Clinical Lung Cancer Genome P, Network Genomic M: Sci Transl Med 2013;5: 209 ra153.

3 Rosell R, Carcereny E, Gervais R, et al.: Lancet Oncol 2012;13:239-246.

4 Mok TS, Wu YL, Thongprasert S, et al.: N Engl J Med 2009;361:947-957.

5 Sequist LV, Yang JC, Yamamoto N, et al.: J Clin Oncol 2013;31:3327-3334.

6 Wu YL, Cheng Y, Zhou X, et al.: Lancet Oncol 2017;18:1454-1466.

7 Soria JC, Ohe Y, Vansteenkiste J, et al.: N Engl J Med 2018;378:113-125.

8 Shaw AT, Kim DW, Nakagawa K, et al.: N Engl J Med 2013;368:2385-2394.

9 Peters S, Camidge DR, Shaw AT, et al.: N Engl J Med 2017;377:829-838.

10 Shaw AT, Kim TM, Crino L, et al.: Lancet Oncol 2017;18:874-886.

11 Kim DW, Tiseo M, Ahn MJ, et al.: J Clin Oncol 2017;35:2490-2498.

12 Solomon BJ, Besse B, Bauer TM, et al.: Lancet Oncol 2018;19:1654-1667.

13 Shaw AT, Ou SH, Bang YJ, et al.: N Engl J Med 2014;371:1963-1971.

14 Planchard D, Smit EF, Groen HJM, et al.: Lancet Oncol 2017;18:1307-1316.

Kontakt: Dr. Matthias Raspe, Dr. Nikolaj Frost, Klinik mit Schwerpunkt Infektiologie und Pneumologie, Charité - Universitätsmedizin Berlin, Campus Virchow Klinikum, Augustenburger Platz 1, 13353 Berlin. 


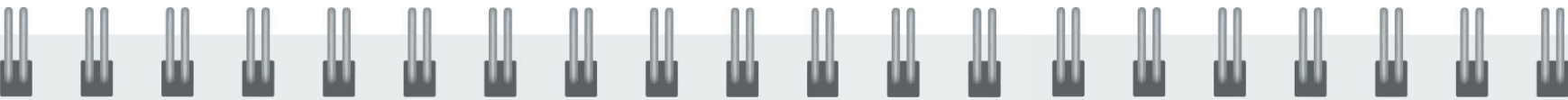

\section{Überlebenstipp für die Weiterbildung}

"Beachten Sie die verschiedenen Abschnitte der Weiterbildung und welche Inhalte in welchen Abschnitten absolviert werden dürfen. So ist beispielsweise die Rotation auf eine Intensivstation erst nach 24 Monaten Weiterbildung anrechenbar. Außerdem sollte die Mindestanzahl an geforderten Rotationen (inklusive definierter Länge) pro Weiterbildungsabschnitt eingehalten werden (sofern die Facharztreife in Regelweiterbildungszeit angestrebt wird). Am Anfang der internistischen Weiterbildung steht die sogenannte Basisweiterbildung von 3 Jahren. Es schließen sich 2 Jahre fachärztliche Weiterbildung zum Internisten oder 3 Jahre fachärztliche Weiterbildung zum Internisten mit Schwerpunkt (2.B. (Facharzt für Innere Medizin und Pneumologie)) an. Natürlich kann auch zunächst der allgemeine Internist (5 Jahre) und anschließend nach 3 weiteren Jahren der Schwerpunktinternist erworben werden- dies bedeutet dann eine Gesamtweiterbildungsdauer von 8 anstatt 6 Jahren. Die Basisweiterbildung beinhaltet ein Jahr sogenannte UUnmittelbare Patientenversorgung) (UPV). Hierunter fallen in der Regel fast alle Fachdisziplinen. Die UPV ermöglicht Quereinsteigern ins Fach die Anrechnung von Weiterbildungszeit aus einem nicht internistischen Fach.»

Matthias Raspe 